\title{
HOSM Controller Using PI Sliding Manifold for an Integrated Active Control for Wheeled Vehicles
}

\author{
Antonio Navarrete Guzmán $\mathbb{D}^{1,},{ }^{1,2}$ Claudia Carolina Vaca García ${ }^{D}{ }^{3}$ \\ Stefano Di Gennaro $\mathbb{D D}^{4,5}$ and Cuauhtémoc Acosta Lúa ${ }^{3,5}$ \\ ${ }^{1}$ Department of Electrical and Electronic Engineering at the National Technological Institute of Mexico Campus Tepic, \\ Av. Tecnológico 2595, Tepic 63175, Nayarit, Mexico \\ ${ }^{2}$ Academic Unit of Basic Sciences and Engineering of the Autonomous University of Nayarit, City of Culture "Amado Nervo", \\ Tepic, Nayarit, Mexico \\ ${ }^{3}$ Departamento De Ciencias Tecnológicas, Universidad De Guadalajara, Centro Universitario De La Ciénega, \\ Av. Universidad 1115, Ocotlán 47820, Jalisco, Mexico \\ ${ }^{4}$ Department of Information Engineering, Computer Science and Mathematics, Via Vetoio, Loc. Coppito, L'Aquila 67100, Italy \\ ${ }^{5}$ Center of Excellence DEWS, University of L'Aquila, Via Vetoio, Loc. Coppito, L'Aquila 67100, Italy
}

Correspondence should be addressed to Cuauhtémoc Acosta Lúa; cuauhtemoc.acosta@cuci.udg.mx

Received 6 April 2021; Revised 6 May 2021; Accepted 8 May 2021; Published 3 June 2021

Academic Editor: Rongwei Guo

Copyright (C) 2021 Antonio Navarrete Guzmán et al. This is an open access article distributed under the Creative Commons Attribution License, which permits unrestricted use, distribution, and reproduction in any medium, provided the original work is properly cited.

\begin{abstract}
This study considers the design of a modified high-order sliding mode (HOSM) controller using a PI sliding surface to the attitude control of a ground vehicle. A robust-modified HOSM controller is derived, so that the lateral velocity and yaw rate tracks the desired trajectory despite the environment actions acting on the ground vehicle and parameter variations. The stability is guaranteed with Lyapunov's stability theorem function. The performance of the dynamic controllers is evaluated using the CarSim simulator considering a challenging double steer maneuver.
\end{abstract}

\section{Introduction}

The integrated active control for wheeled vehicles is an important topic due to improving the drivability and safety of the vehicle in a critical situation. This active control employs electronic actuators in the vehicles. The new actuators offer great flexibility when a design control architecture allows the controller to be designed as a separate block. This decoupling helps to design the controller as an active front steering (AFS) or rear torque vectoring (RTV). The AFS controller imposes a steering angle to correct the action of the driver. The RTV controller imposes an active action in the yaw momentum of the vehicle. All these control actions render more safety to the ground vehicle and passengers.

The control action is usually determined using approximation models because they capture the main aspect of the physics of the problem. But these mathematical models can be extended due to the presence of parameter uncertainties/variations and the presence of disturbances acting on the vehicle, among which are the change of parameters due to the road condition and the environmental disturbance acting on the vehicle (front and lateral wind). On this subject, the reader can find in [1-4]. However, many other mathematical models that include uncertainty in the parameters and external disturbances are available in the literature and could be considered, for instance, those in $[5,6]$.

The article presents the combination of the modified HOSM with a PI sliding surface. The principal idea is to design a particular sliding surface on which the system dynamics are constrained to evolve by the modified HOSM. It is noted that the controller presented here maintains the inherent properties of the HOSM, such as robustness. For the above reasons, this article deals with designing a modified high-order sliding mode (HOSM) controller using a PI sliding surface to track the desired trajectory for lateral 
velocity and angular references despite variations in some parameters and external disturbances such as wind gusts. For this purpose, the active front steering (AFS) and rear torque vectoring (RTV) are considered as the integrated active control, and the aim is to improve the performance of the control system applied in automobiles. The proposed controller uses all the information from sensors that are currently available in modern vehicles. The closed-loop system stability has been proven in the sense of Lyapunov through the development of the candidate function. It was considered that the CarSim simulator to validate the controller is proposed. CarSim is a software that predicts dynamic vehicle behavior. In fact, CarSim delivers the most accurate, detailed, and efficient methods for simulating the performance of passenger vehicles and is supported by automotive enterprises, such as the Ford Motor Company and Chrysler.

Earlier research on the integrated active controller is based on the nonlinear techniques as [7-9], and for more recent results, [10-14]. In [15], a nonlinear decoupling control approach is presented for a three-degree of freedom model. In [16], a yaw-stabilizing algorithm is presented, combining AFS with a low-level control of the longitudinal wheel slip, with adaptive yaw estimating the maximum tireroad friction parameter for each wheel. In [17], AFS and RTV are combined in an integrated controller to guarantee vehicle stability, making use of adaptive feedback.

The HOSM was introduced in [18], and the main advantage of the high-order sliding mode is to overcome the chattering problem. A further notable advantage of HOSM is the possibility of considering sliding mode surfaces with a relative degree greater than one [19] and has great advantages compared to the sliding mode [20]. However, to add the integral action in discontinuous controllers as in the case of first-order sliding modes to improve the chattering phenomenon and smoothness of the controller action; the chattering is caused by reaching the sliding surface in finite time asymptotically fast, and this behavior generates the actuator commute quickly [21]. In the case of HOSM, adding a discontinuous integral action eliminates the effect of the disturbance; considering this action as an estimator of the disturbance [22], it has been shown that to continue adding integrators helps to decrease the chattering effect. Nonetheless, there are studies in which the integral action is added on the surface, improving the behavior of the controller in the transient time.

Nevertheless, the reader can find works in which HOSM uses a PI controller as the sliding surface. In [23], the PID sliding mode control scheme is designed for a high-speed train (HST) subjected to actuator faults, asymmetric nonlinear actuator saturation. A combination of SMC with PID sliding surface is used to control a 2-degree of freedom (2DOF) planar manipulator [24]. The study by Gu et al. [25] presents an SMC-PI sliding surface for robust tracking control of a nanopositioning stage composed of piezoceramic stack actuators (PSAs) and compliant flexure mechanisms. A second-order sliding mode using PI sliding surface applied in the control of DC motor drive is used in [26]. In [27], a sliding mode control with PID sliding surface is used to activate vibration damping of pneumatically actuated soft robots. In [28], the supertwisting algorithm combined with the proportional integral derivative sliding mode control modified is designed to solve trajectory tracking and stabilize the quadrotor attitude.

The study is organized as follows. In Section 2, the mathematical model of a vehicle is recalled, and the control problem is formulated. In Section 3, a modified HOSM with the PI sliding surface controller is presented. In Section 4, the resulting controller is tested with CarSim simulations. Some comments conclude the study.

\section{Mathematical Model of the Vehicle Dynamics}

In this article, to obtain the mathematical model of the ground vehicle, it is considered as a rigid body connected to the ground through the tires. This model is the so-called bicycle model or single-track model $[29,30]$ and is widely used by scientists to design nonlinear control due to preserving the essence of basic vehicle dynamics because it uses only longitudinal/lateral and yaw dynamics. The effectiveness of the bicycle model will be validated into a virtual automobile software (CarSim simulator) where it is testing the controller performance. In $[31,32]$, the active front steering (AFS) is considered as an input control; this actuator will impose an incremental steer angle $\delta_{c}$. On the other hand, it also considered the yaw momentum as a control input $M_{z}$, which imposes negative longitudinal forces using the active brakes. Hence, the mathematical model of the ground vehicle is

$$
\begin{aligned}
m\left(\dot{v}_{x}-v_{y} \omega_{z}\right) & =\mu_{x}\left(F_{x, f}+F_{x, r}\right)+F_{d, x}, \\
m\left(\dot{v}_{y}+v_{x} \omega_{z}\right) & =\mu_{y}\left(F_{y, f}+F_{y, r}\right)+F_{d, y}, \\
J_{z} \dot{\omega}_{z} & =\mu_{y}\left(l_{f} F_{y, f}-l_{r} F_{y, r}\right)+M_{z}+M_{d, z},
\end{aligned}
$$

where $v_{x}$ and $v_{y}$ are the longitudinal and lateral velocities of the vehicle center of mass, and $\alpha_{z}$ and $\omega_{z}$ are the yaw angle and yaw rate of the vehicle, $m$ is the vehicle mass, $J_{z}$ is the inertia momentum, $l_{f}$ is the distance between the center mass to the front wheels, and $l_{r}$ is the distance between the center mass to the rear wheels. Also, $\mu_{x}$ and $\mu_{y}$ are the longitudinal/lateral tire-road friction coefficients. Moreover, $F_{x, f}$ and $F_{x, r}$ are the front/rear longitudinal forces and $F_{y, f}$ and $F_{y, r}$ are the rear longitudinal/lateral forces, and $M_{z}$ is the yaw moment. The external disturbance forces, due to environment actions as the wind blast, acting on the vehicle dynamics are $F_{d, x}$ and $F_{d, y}$ and $M_{d, z}$ is the torque generated by the external disturbance.

The tire front lateral force $F_{y, f}$ depends directly the front tire slip angle $\alpha_{f}=\delta_{d}+\delta_{c}-\left(\left(v_{y}+l_{f} \omega_{z}\right) / v_{x}\right)$, where $\delta_{d}$ is the road wheel angle imposed to the drive and the $\delta_{c}$ is the active front steering imposed by the designed control. Model (1) presents nonlinearities due to the characteristics of the tires. In this article, is used the compact Pacejka magic formula:

$$
F_{y, j}\left(\alpha_{j}\right)=D_{j} \sin \left[C_{j} \arctan \left(B_{j} \alpha_{j}\right)\right], \quad j=f, r,
$$

where $B_{j}, C_{j}$, and $D_{j}$ are the values obtained experimentally [33]. 
The tire slip angle $\alpha_{j}$, which depends all the forces acting in the vehicle dynamics, has some properties such as the angle has a minimum $/$ maximum value, i.e., $\alpha_{j}= \pm \alpha_{j, \max }$, and it is considered invertible in function to $\alpha_{j}$ : $\alpha_{j} \in\left[-\alpha_{j, \max }, \alpha_{j, \max }\right]$.

The tire front lateral force, $F_{y, f}\left(\delta_{d}+\delta_{c}, v_{y}, \omega_{z}\right)$, is considered an invertible function with respect to the AFS control input $\delta_{c}$; therefore, for a fixed value $F_{y, f, 0}$, the solution of $F_{y, f}\left(\delta_{d}+\delta_{c}, v_{y}, \omega_{z}\right)=F_{y, f, 0}$ is unique and given by

$$
\delta_{c}= \begin{cases}-\delta_{d}+\frac{v_{y}+l_{f} \omega_{z}}{v_{x}}+F_{y, f}^{-1}\left(F_{y, f, 0}\right), & \text { if } \alpha_{f} \in\left[-\alpha_{f, \max }, \alpha_{f, \max }\right], \\ -\delta_{d}+\frac{v_{y}+l_{f} \omega_{z}}{v_{x}} \pm F_{y, f, \max }, & \text { otherwise. }\end{cases}
$$

The lateral force $F_{y, f}\left(\delta_{d}+\delta_{c}, v_{y}, \omega_{z}\right)$ is considered an invertible function; for this reason, it is possible to consider the following control input:

$$
\Delta_{f}=F_{y, f}\left(\delta_{d}+\delta_{c}, v_{y}, \omega_{z}\right)-F_{y, f}\left(\delta_{d}, v_{y}, \omega_{z}\right) .
$$

Substituting $\Delta_{f}$ controller into the lateral velocity and the yaw rate velocity in the mathematical model of the ground vehicle (1), besides $F_{x, f}+F_{x, r}=0$, the vehicle dynamics is rewritten as

$$
\begin{aligned}
& \dot{v}_{x}=v_{y} \omega_{z}+\frac{1}{m} F_{d, x}, \\
& \dot{v}_{y}=-v_{x} \omega_{z}+\frac{\mu_{y}}{m}\left(F_{y, f}\left(\delta_{d}\right)+F_{y, r}\right)+\frac{\mu_{y}}{m} \Delta_{f}+\frac{1}{m} F_{d, y}, \\
& \dot{\omega}_{z}=\frac{\mu_{y}}{J_{z}}\left(l_{f} F_{y, f}\left(\delta_{d}\right)-l_{r} F_{y, r}\right)+\frac{\mu_{y} l_{f}}{J_{z}} \Delta_{f}+\frac{1}{J_{z}} M_{z}+\frac{1}{J_{z}} M_{d, z} .
\end{aligned}
$$

The control aim is to design a modified HOSM controller using PI sliding surface, such as the lateral velocity $v_{y}$ that globally tracks a reference $v_{y \text {,ref }}$, and $\omega_{z}$ tends a reference yaw rate $\omega_{z \text {,ref }}$ in finite time, despite the presence of the external disturbances and parameter uncertainties.
In this article, it is considered that the longitudinal velocity $v_{x}$, the lateral velocity $v_{y}$ and the yaw rate $\omega_{z}$ are measurable variables. To solve the control problem, the following assumptions will be used.

Assumption 1. The steer angle $\delta_{d}$ is considered a smooth function, i.e., function $C^{2}$.

Assumption 2. The variables of the reference $v_{y \text {,ref }}$ and $\omega_{z \text {,ref }}$ and their derivatives $\dot{v}_{y \text {,ref }}$ and $\dot{\omega}_{z \text {,ref }}$ are considered bounded (physically obvious).

\section{Design a Modified HOSM Controller for Trajectory Tracking}

In this section, a modified HOSM controller using PI sliding surface will be designed when the parameter uncertainties and external disturbances are known.

Consider the tracking error as

$$
\begin{aligned}
& e_{v, y}=v_{y}-v_{y, \text { ref }}, \\
& e_{\omega, z}=\omega_{z}-\omega_{z, \text { ref }} .
\end{aligned}
$$

and the dynamics of the error system is

$$
\begin{aligned}
& \dot{e}_{v, y}=-v_{x} \omega_{z}+\frac{\mu_{y}}{m}\left(F_{y, f}\left(\delta_{d}\right)+F_{y, r}\right)+\frac{\mu_{y}}{m} \Delta_{f}+\frac{1}{m} F_{d, y}-\dot{v}_{y, \text { ref }}, \\
& \dot{e}_{\omega, z}=\frac{\mu_{y}}{J_{z}}\left(l_{f} F_{y, f}\left(\delta_{d}\right)-l_{r} F_{y, r}\right)+\frac{\mu_{y} l_{f}}{J_{z}} \Delta_{f}+\frac{1}{J_{z}} M_{z}+\frac{1}{J_{z}} M_{d, z}-\dot{\omega}_{z, \mathrm{ref}} .
\end{aligned}
$$

Using the tracking error (6), the PI sliding function is defined as

$$
\begin{aligned}
& s_{y}=k_{p, y} e_{v, y}(t)+k_{i, y} \int e_{v, y}(t) \mathrm{d} t, \\
& s_{z}=k_{p, z} e_{\omega, z}(t)+k_{i, z} \int e_{\omega, z}(t) \mathrm{d} t,
\end{aligned}
$$

where $k_{p, y}, k_{i, y}, k_{p, z}, k_{i, z}>0$. The gains in (8) provide flexibility for the construction of the PI sliding surface.

Then, it is possible to obtain the derivative with respect to time of the PI sliding function (8) as

$$
\begin{aligned}
& \dot{s}_{y}=k_{p, y}\left(-v_{x} \omega_{z}+\frac{\mu_{y}}{m}\left(F_{y, f}\left(\delta_{d}\right)+F_{y, r}\right)+\frac{\mu_{y}}{m} \Delta_{f}+\frac{1}{m} F_{d, y}-\dot{v}_{y, \mathrm{ref}}\right)+k_{i, y} e_{v, y}, \\
& \dot{s}_{z}=k_{p, z}\left(\frac{\mu_{y}}{J_{z}}\left(l_{f} F_{y, f}\left(\delta_{d}\right)-l_{r} F_{y, r}\right)+\frac{\mu_{y} l_{f}}{J_{z}} \Delta_{f}+\frac{1}{J_{z}} M_{z}+\frac{1}{J_{z}} M_{d, z}-\dot{\omega}_{z, \mathrm{ref}}\right)+k_{i, z} e_{\omega_{z}} .
\end{aligned}
$$

Hence, taking into account the PI surface $\dot{s}_{y}(9)$, the following input control $\Delta_{f}$ is proposed:

$$
\begin{aligned}
\Delta_{f}= & -\frac{m}{\mu_{y}}\left(-v_{x} \omega_{z}+\frac{\mu_{y}}{m}\left(F_{y, f}\left(\delta_{d}\right)+F_{y, r}\right)+\frac{1}{m} F_{d, y}-\dot{v}_{y, \text { ref }}\right) \\
& -\frac{m}{\mu_{y}} \frac{k_{i, y}}{k_{p, y}} e_{v, y}+\frac{m}{\mu_{y} k_{p, y}}\left(-\lambda_{1, y}\left|s_{y}\right|^{(1 / 2)} \operatorname{sign}\left(s_{y}\right)-\lambda_{2, y} s_{y}+\int_{0}^{t}\left(-\lambda_{3, y}\right) \operatorname{sign}\left(s_{y}(\tau)\right) \mathrm{d} \tau+\int_{0}^{t}\left(-\lambda_{4, y}\right) s_{y}(\tau) \mathrm{d} \tau\right),
\end{aligned}
$$


where $\lambda_{1, y}, \lambda_{2, y}, \lambda_{3, y}, \lambda_{4, y}>0$.

Similarly, the RTV controller takes into account $\dot{s}_{z}(9)$, and proposing the controller $M_{z}$ as

$$
\begin{aligned}
M_{z}=- & J_{z}\left(\frac{\mu_{y}}{J_{z}}\left(l_{f} F_{y, f}\left(\delta_{d}\right)-l_{r} F_{y, r}\right)+\frac{\mu_{y} l_{f}}{J_{z}} \Delta_{f}+\frac{1}{J_{z}} M_{d, z}-\dot{\omega}_{z, \mathrm{ref}}\right) \\
& \quad-J_{z} \frac{k_{i, z}}{k_{p, z}} e_{\omega_{z}}+\frac{J_{z}}{k_{p, z}}\left(-\lambda_{1, z}\left|s_{z}\right|^{(1 / 2)} \operatorname{sign}\left(s_{z}\right)-\lambda_{2, z} s_{z}+\int_{0}^{t}\left(-\lambda_{3, z}\right) \operatorname{sign}\left(s_{z}(\tau)\right) \mathrm{d} \tau+\int_{0}^{t}\left(-\lambda_{4, z}\right) s_{z}(\tau) \mathrm{d} \tau\right),
\end{aligned}
$$

where $\lambda_{1, z}, \lambda_{2, z}, \lambda_{3, z}, \lambda_{4, z}>0$.

Substituting the input control (10) into the PI sliding surface $\dot{s}_{y}$ in (9), one obtains

$$
\begin{aligned}
& \dot{s}_{y}=-\lambda_{1, y}\left|s_{y}\right|^{(1 / 2)} \operatorname{sign}\left(s_{y}\right)-\lambda_{2, y} s_{y}+\chi_{y}, \\
& \dot{\chi}_{y}=-\lambda_{3, y} \operatorname{sign}\left(s_{y}\right)-\lambda_{4, y} s_{y},
\end{aligned}
$$

and the input control $M_{z}(11)$ into the PI sliding surface $\dot{s}_{z}$, one obtains

$$
\begin{aligned}
& \dot{s}_{z}=-\lambda_{1, z}\left|s_{z}\right|^{(1 / 2)} \operatorname{sign}\left(s_{z}\right)-\lambda_{2, z} s_{z}+\chi_{z}, \\
& \dot{\chi}_{z}=-\lambda_{3, z} \operatorname{sign}\left(s_{z}\right)-\lambda_{4, z} s_{z} .
\end{aligned}
$$

In fact, from (12) and (13), one gets the following differential inclusion:

$$
\begin{aligned}
& \dot{s}_{j}=-\lambda_{1, j} s_{j}{ }^{(1 / 2)}-\lambda_{2, j} s_{j}+\chi_{j}, \\
& \dot{\chi}_{j}=-\lambda_{3, j} s_{j}{ }^{0}-\lambda_{4, j} s_{j},
\end{aligned}
$$

where $j=s, z$, and $s_{j}{ }^{(1 / 2)}=\left|s_{j}\right|^{(1 / 2)} \operatorname{sign}\left(s_{j}\right)$ and $s_{j}{ }^{0}=\operatorname{sign}$ $\left(s_{j}\right)$.

$$
\begin{aligned}
\frac{\mathrm{d}}{\mathrm{d} t} s_{j}{ }^{(1 / 2)} & =\frac{1}{2\left|s_{j}\right|^{(1 / 2)}}\left(-\lambda_{1, j} s_{j}{ }^{(1 / 2)}-\lambda_{2, j} s_{j}+\chi_{j}\right)=-\frac{1}{2\left|s_{j}\right|^{(1 / 2)}}\left(\lambda_{1, j} s_{j}{ }^{(1 / 2)}-\chi_{j}\right)-\frac{1}{2} \lambda_{2, j} s_{j}{ }^{(1 / 2)}, \\
\dot{s}_{j} & =-\lambda_{1, j} s_{j}{ }^{(1 / 2)}-\lambda_{2, j} s_{j}+\chi_{j}, \\
\dot{\chi}_{j} & =-\lambda_{3, j} s_{j}{ }^{0}-\lambda_{4, j} s_{j}=-\frac{1}{2\left|s_{j}\right|^{(1 / 2)}} 2 \lambda_{3, j} s_{j}{ }^{(1 / 2)}-\lambda_{4, j} s_{j} .
\end{aligned}
$$

The derivative of $(15)$ is

$$
\dot{\mathscr{V}}=\xi^{T} P \dot{\xi},
$$

$$
\dot{\xi}=\left(\begin{array}{c}
s_{j}^{(1 / 2)} \\
\dot{s}_{j} \\
\dot{\chi}_{j}
\end{array}\right)=-\frac{1}{2\left|\dot{s}_{j}\right|^{(1 / 2)}} \Lambda_{1} \xi+\frac{1}{2} \Lambda_{2} \xi,
$$

with
The proof of the stability of the origin to the PI sliding surface $s_{j}$ can be considered as the following Lyapunov function

$$
\begin{gathered}
\mathscr{V}=\frac{1}{2} \xi^{T} P \xi, \\
\lambda_{\min }^{P}\|\xi\|_{2}^{2} \leq \mathscr{V}(\xi) \leq \lambda_{\text {max }}^{P}\|\xi\|_{2}^{2},
\end{gathered}
$$

where $\mathscr{V}$ is continuous and differentiable when $s_{j} \neq 0$, and

$$
\begin{aligned}
& \xi=\left(\begin{array}{c}
s_{j}{ }^{(1 / 2)} \\
s_{j} \\
\chi_{j}
\end{array}\right), \\
& P=\left(\begin{array}{ccc}
\lambda_{1, j}^{2}+4 \lambda_{3, j} & \lambda_{1, j} \lambda_{2, j} & -\lambda_{1, j} \\
\lambda_{1, j} \lambda_{2, j} & 2 \lambda_{4, j}+\lambda_{2, j}^{2} & -\lambda_{2, j} \\
-\lambda_{1, j} & -\lambda_{2, j} & 2
\end{array}\right) .
\end{aligned}
$$

Let $s_{j}=\left|s_{j}\right|^{(1 / 2)} s_{j}{ }^{(1 / 2)}$ and $s_{j}{ }^{0}=\left(1 / 2\left|s_{j}\right|^{(1 / 2)}\right) 2 s_{j}{ }^{(1 / 2)}$, the derivative of $\xi$ with respect to time will be 


$$
\begin{aligned}
\dot{\mathscr{V}}= & -\frac{1}{2\left|s_{j}\right|^{(1 / 2)} \xi^{T} P \Lambda_{1} \xi+\frac{1}{2} \xi^{T} P \Lambda_{2} \xi}, \\
P \Lambda_{1}= & \left(\begin{array}{ccc}
\lambda_{1, j}\left(\lambda_{1, j}^{2}+2 \lambda_{3, j}\right) & 0 & -\lambda_{1, j}^{2} \\
\lambda_{2, j}\left(\lambda_{1, j}^{2}-2 \lambda_{3, j}\right) & 0 & -\lambda_{1, j} \lambda_{2, j} \\
-\lambda_{1, j}^{2} & 0 & \lambda_{1, j}
\end{array}\right), \\
P \Lambda_{2}= & -\left(\begin{array}{ccc}
-\lambda_{2, j}\left(-3 \lambda_{1, j}^{2}+4 \lambda_{3, j}\right) & -2 \lambda_{1, j}\left(\lambda_{2, j}^{2}-\lambda_{4, j}\right) & 2 \lambda_{1, j} \lambda_{2, j} \\
-\lambda_{1, j}\left(3 \lambda_{2, j}^{2}+4 \lambda_{4, j}\right) & -2 \lambda_{2, j}\left(\lambda_{2, j}^{2}+\lambda_{4, j}\right) & \left(2 \lambda_{2, j}^{2}+4 \lambda_{4, j}\right) \\
3 \lambda_{1, j} \lambda_{2, j} & \left(2 \lambda_{2, j}^{2}-4 \lambda_{4, j}\right) & -2 \lambda_{2, j}
\end{array}\right) .
\end{aligned}
$$

The first term of (20), i.e., $-\left(1 / 2\left|s_{j}\right|^{(1 / 2)}\right) \xi^{T} P \Lambda_{1} \xi$ can be rewritten as

$$
-\frac{1}{2\left|s_{j}\right|^{1 / 2}} \xi^{T} P \Lambda_{1} \xi=-\frac{1}{2\left|s_{j}\right|^{(1 / 2)}}\left(\lambda_{1, j}\left(2 \lambda_{3, j}+\lambda_{1, j}^{2}\right)\left(s_{j}{ }^{(1 / 2)}\right)^{2}-2 \lambda_{1, j}^{2} s_{j}^{(1 / 2)} x_{j}+\lambda_{2, j}\left(\lambda_{1, j}^{2}-2 \lambda_{3, j}\right) s_{j}{ }^{(1 / 2)} s_{j}-\lambda_{1, j} \lambda_{2, j} s_{j} x_{j}+\lambda_{1, j} x_{j}^{2}\right)
$$

if $-\left(1 / 2\left|s_{j}\right|{ }^{(1 / 2)}\right) \lambda_{2, j} \quad\left(\lambda_{1, j}^{2}-2 \lambda_{3, j}\right) s_{j}{ }^{(1 / 2)} s_{j}=-(1 / 2) \lambda_{2, j}$ $\left(\lambda_{1, j}^{2}-2 \lambda_{3, j}\right)\left(s_{j}{ }^{(1 / 2)}\right)^{2}$, the first term in matrix form is written as

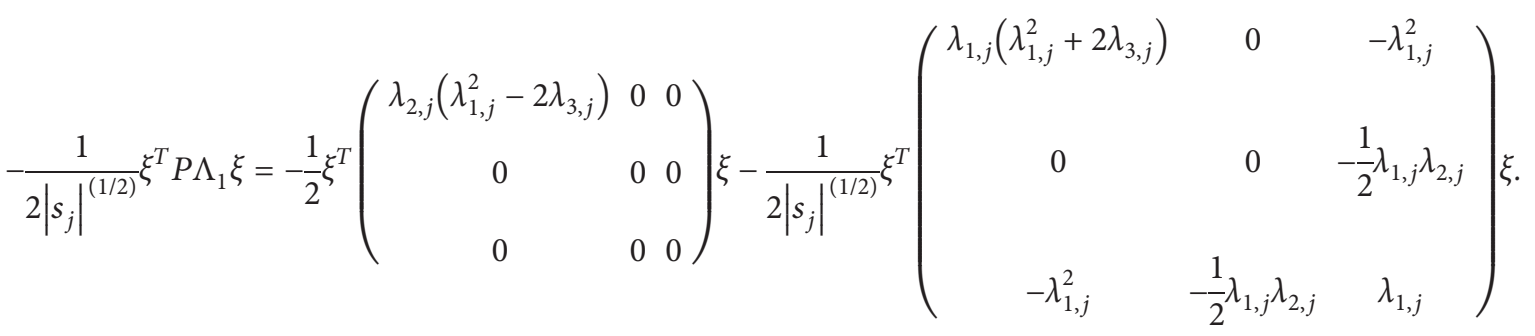

Similarly, the second term of (20) is $(1 / 2) \xi^{T} P \Lambda_{2} \xi$, and it is written as

$$
\frac{1}{2} \xi^{T} P \Lambda_{2} \xi=\frac{1}{2}\left(-\lambda_{2, j}\left(3 \lambda_{1, j}+4 \lambda_{3, j}\right)\left(s_{j}{ }^{(1 / 2)}\right)^{2}-2 \lambda_{2, j}\left(\lambda_{2, j}+\lambda_{4, j}\right) s_{j}^{2}-2 \lambda_{2, j} x_{j}^{2}-\lambda_{1, j}\left(5 \lambda_{2, j}^{2}+2 \lambda_{4, j}\right) s_{j}{ }^{(1 / 2)} s_{j}+5 \lambda_{1, j} \lambda_{2, j} s_{j}{ }^{(1 / 2)} x_{j}+4 \lambda_{2, j}^{2} s_{j} x_{j}\right),
$$

since $s_{j}{ }^{(1 / 2)} s_{j}=\left(1 / 2\left|s_{j}\right|^{(1 / 2)}\right) 2 s_{j}^{2}$ and $s_{j}{ }^{(1 / 2)} x_{j}=\left(1 /\left|s_{j}\right|^{(1 / 2)}\right)$ $s_{j} x_{j}$, the second term in matrix form is 


$$
\begin{aligned}
& \frac{1}{2} \xi^{T} P \Lambda_{2} \xi=-\frac{1}{2} \lambda_{2, j} \xi^{T}\left(\begin{array}{ccc}
3 \lambda_{1, j}^{2}+4 \lambda_{3, j} & 0 & 0 \\
0 & 2\left(\lambda_{2, j}^{2}+\lambda_{4, j}\right) & -2 \lambda_{2, j} \\
0 & -2 \lambda_{2, j} & 2
\end{array}\right) \xi-\frac{1}{2\left|s_{j}\right|^{(1 / 2)}} \lambda_{1, j} \xi^{T}\left(\begin{array}{cc}
0 & 0 \\
0 & 5 \lambda_{2, j}^{2}+2 \lambda_{4, j} \\
-\frac{5}{2} \lambda_{2, j} \\
0 \\
0
\end{array}\right) \xi \\
& \dot{V}_{v, \kappa}=-\frac{k_{i, \kappa}}{k_{p, \kappa}} e_{v, \kappa}^{2}(t) \mathrm{d} t,
\end{aligned}
$$

$$
\dot{\mathscr{V}}=-\frac{1}{\left|s_{j}\right|^{(1 / 2)}} \xi^{T} Q_{1} \xi-\xi^{T} Q_{2} \xi,
$$

with

$$
\begin{aligned}
& Q_{1}=\frac{\lambda_{1, j}}{2}\left(\begin{array}{ccc}
\left(\lambda_{1, j}^{2}+2 \lambda_{3, j}\right) & 0 & -\lambda_{1, j} \\
0 & 2 \lambda_{4, j}+5 \lambda_{2, j}^{2} & -3 \lambda_{2, j} \\
-\lambda_{1, j} & -3 \lambda_{2, j} & 1
\end{array}\right), \\
& Q_{2}=\frac{\lambda_{2, j}}{2}\left(\begin{array}{ccc}
2 \lambda_{1, j}^{2}+\lambda_{3, j} & 0 & 0 \\
0 & \lambda_{2, j}^{2}+\lambda_{4, j} & -\lambda_{2, j} \\
0 & -\lambda_{2, j} & 1
\end{array}\right),
\end{aligned}
$$

where $\dot{\mathscr{V}}$ is the negative definitive if and only if the matrix $Q_{1}, Q_{2}>0$, namely, $\lambda_{1, j}, \lambda_{2, j}, \lambda_{3, j}, \lambda_{4, j}>0$. Denoting by $\lambda_{\text {min }}^{Q_{1}}, \lambda_{\text {min }}^{Q_{2}}$ the minimum eigenvalues of the matrix $Q_{1}, Q_{2}$, one finally works out

$$
\dot{\mathscr{V}} \leq \frac{1}{\left|s_{j}\right|^{(1 / 2)}} \lambda_{\min }^{Q_{1}}\|\xi\|_{2}^{2}-\lambda_{\min }^{Q_{2}}\|\xi\|_{2}^{2},
$$

and using the fact $\left|e_{v}\right|^{(1 / 2)} \leq\|\xi\|_{2} \leq \sqrt{\left(2 \mathscr{V} / \lambda_{\min }^{P}\right)}$, one obtains

$$
\dot{\mathscr{V}} \leq-\left(c_{1} \mathscr{V}^{(1 / 2)}+c_{2} \mathscr{V}\right)
$$

where $c_{1}=\sqrt{2 \lambda_{\min }^{P}}\left(\lambda_{\min }^{Q_{1}} / \lambda_{\max }^{P}\right)$ and $c_{2}=2\left(\lambda_{\min }^{Q_{2}} / \lambda_{\max }^{P}\right)$.

\section{Sliding Modes Dynamics Stability}

To determinate the stability of the sliding surface in (8), we focus when the sliding surface occurs, $s_{i}=0 ; i=y, z$, then

$$
\begin{aligned}
s_{i} & =k_{p, i} e_{v, \kappa}(t)+k_{i, \kappa} \int e_{v, \kappa}(t) \mathrm{d} t, \quad \kappa=y, z, \\
e_{v, \kappa}(t) & =-\frac{k_{i, \kappa}}{k_{p, \kappa}} \int e_{v, \kappa}(t) \mathrm{d} t .
\end{aligned}
$$

For the analysis of stability of error (29), the Lyapunov candidate function is proposed as

$$
V_{v, \kappa}=\frac{1}{2} e_{v, \kappa}(t)^{2}
$$

The dynamic of the Lyapunov function yields
Therefore, the error $e_{v, k}(t)$ is asymptotically stable when the gains $k_{i, \kappa}>0$ and $k_{p, \kappa}>0$.

\section{Simulation Results}

In this section, the simulation results taking into account the mathematical model of the vehicle (1) and the controllers (10) and (11) are presented using the CarSim simulator. CarSim delivers the most accurate, detailed, and efficient methods for simulating the performance of passenger vehicles. The test maneuver considered in this simulation is a so-called double-step steer (Figure 1). The maneuver consists of a rapid turn on the left of $100^{\circ}$ at $t=1 \mathrm{~s}$, followed by a turn on the right of $-100^{\circ}$ at $t=3 \mathrm{~s}$, and finally, at $t=5 \mathrm{~s}$, the steering wheel is set to zero. The parameters considered in (1) are given in Table 1.

The reference variables $v_{y, \text { ref }}(t)$ and $\omega_{z \text {,ref }}(t)$ will be considered as the behavior of an "ideal" or "reference" vehicle.

$$
\begin{aligned}
& \dot{v}_{y, r}=-v_{x} \omega_{z, \mathrm{ref}}+\frac{\mu_{y, r}}{m_{r}}\left(F_{y, f, r}\left(\alpha_{f, r}\right)+F_{y, r, r}\left(\alpha_{r, r}\right)\right), \\
& \dot{\omega}_{z, r}=\frac{\mu_{y, r}}{J_{z, r}}\left(F_{y, f, r}\left(\alpha_{f, r}\right) l_{f, r}-F_{y, r, r}\left(\alpha_{r, r}\right) l_{r, r}\right),
\end{aligned}
$$

where $\alpha_{f, r}=\delta_{d}-\left(\left(v_{y, \text { ref }}+l_{f, r} \omega_{z, \text { ref }}\right) / v_{x}\right), \alpha_{r, r}=\left(-\left(v_{y, \text { ref }}-\right.\right.$ $\left.\left.l_{r, r} \omega_{z, \text { ref }}\right) / v_{x}\right)$, and the tire-road friction coefficient reference $\mu_{y, r}$, which is assumed as the road, is dry. The reference forces $F_{y, f, r}$ and $F_{y, r, r}$ are obtained using the compact Pacejka magic formula (2). The parameters considered in (32) are given in Table 2.

Finally, a disturbance due to the wind is considered, which has been implemented in $[2,4]$. The wind induces longitudinal/lateral forces $F_{d, x}, F_{d, y}$, and a yaw moment $M_{d, z}$ [34]. In terms of the front/lateral surfaces $A_{s, f}, A_{s, l}$ of the vehicle, the air density $\rho$, and the (dimensionless) front/ lateral aerodynamic coefficients $c_{a, x}, c_{a, y}$, the expression of the forces, and yaw torque due to the wind are $F_{d, x}=-\left(A_{s, f} \rho c_{a, x} v_{a w, x}^{2} / 2\right), \quad F_{d, y}=-\left(A_{s, l} \rho c_{a, y} v_{a w, y}^{2} / 2\right)$, $M_{d, z}=l_{c} F_{d, y}$, where $l_{c}=l_{c 0}+0.025 \mathcal{N}$ is the distance between the center of mass and the center of pressure, with $l_{c 0}$ the nominal value and $\mathcal{N}$ a white noise. The parameters considered are given in Table 3.

5.1. CarSim Simulations. The simulations consider the performance of the controllers (10) and (11). The model of the vehicle considered in the CarSim simulator is a C-class hatchback automobile. The tires selected are 245/40-R17. 


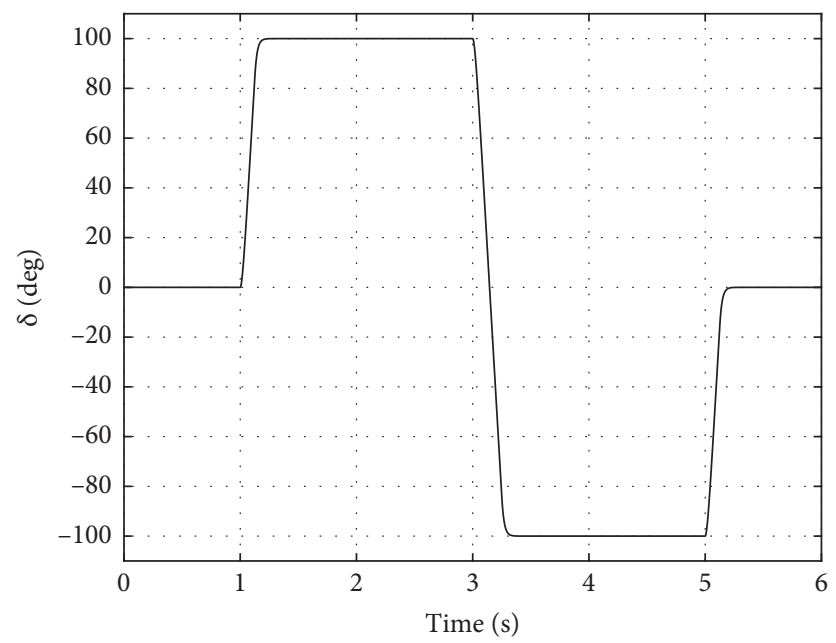

Figure 1: Steering wheel angle $\delta_{d}$.

TABLE 1: Nominal parameter values.

\begin{tabular}{lcc}
\hline$m_{0}=1565 \mathrm{~kg}$ & $B_{f, 0}=16$ & $C_{f, 0}=1.41$ \\
$J_{z, 0}=2075 \mathrm{kgm}^{2}$ & $B_{r, 0}=16$ & $C_{r, 0}=1.51$ \\
Steer ratio: 16.01 & $D_{f, 0}=8854 \mathrm{~N}$ & $E_{f, 0}=0$ \\
$l_{f}=1.38 \mathrm{~m}$ & $D_{r, 0}=8394 \mathrm{~N}$ & $E_{r, 0}=0$ \\
$l_{r}=1.53 \mathrm{~m}$ & - &
\end{tabular}

TABLE 2: Constants of the reference system (32).

\begin{tabular}{lrr}
\hline$m_{r}=1862 \mathrm{~kg}$ & $B_{y, f, r}=B_{y, f}$ & $D_{y, f, r}=10000 \mathrm{~N}$ \\
$l_{f, r}=l_{f}$ & $B_{y, r, r}=14.7$ & $D_{y, r, r}=10000 \mathrm{~N}^{2}$ \\
$l_{r, r}=l_{r}$ & $C_{y, f, r}=C_{y, f}$ & $J_{z, r}=2488 \mathrm{kgm}^{2}$ \\
$\mu_{y, r}=0.9$ & $C_{y, r, r}=1.2$ & - \\
\hline
\end{tabular}

TABle 3: Parameters of the external disturbance.

\begin{tabular}{lcr}
\hline$\mu_{y d}=0.9$ & $A_{s, f}=2.59 \mathrm{~m}^{2}$ & $c_{a, x}=0.3$ \\
$\mu_{y w}=0.6$ & $A_{s, l}=5.1 \mathrm{~m}^{2}$ & $c_{a, y}=0.6$ \\
$\mu_{x}=\mu_{y}$ & $l_{c 0}=-0.20 \mathrm{~m}$ & $\rho=1.2 \mathrm{~kg} / \mathrm{m}^{3}$ \\
\hline
\end{tabular}

The vehicle concerned has independent suspensions on both axles. The nominal values $m_{0}, J_{z, 0}, D_{f, 0}, D_{r, 0}$, and $l_{c 0}$ have been used in the controller (Table 1), while the real automobile parameters are $m=1.15 m_{0}, J=1.15 J_{z, 0}$, $D_{f}=0.85 D_{f, 0}$, and $D_{r}=0.85 D_{r, 0}$. The variables considered measurables in the CarSim are the longitudinal/ lateral velocities $v_{x}$ and $v_{y}$ and the yaw angle and rate $\alpha_{z}$ and $\omega_{z}$. The inputs to the CarSim simulator are the steering angle $\delta=\delta_{d}+\delta_{c}$, with $\delta_{c}$ calculated using (3), and the yaw moment $M_{z}$, and other inputs are the wind (windheading and wind-speed) and longitudinal/lateral tireroad.
The initial conditions used to show the performance of the controllers (10) and (11) are $v_{x}(0)=100 \mathrm{~km} / \mathrm{h}, v_{y}(0)=0$ $\mathrm{km} / \mathrm{h}, \omega_{z}(0)=0 \mathrm{deg} / \mathrm{s}$, and $\alpha_{z}(0)=0 \mathrm{deg}$, and the simulation results are shown in Figures 1-7.

The longitudinal/lateral tire-road friction coefficients $\mathrm{mu}_{x}$ and $\mathrm{mu}_{y}$ are given in Figure 2(a), and the side-slip angle behavior $\beta=\left(v_{y} / v_{x}\right)$ is given in Figure 2(b). The control input $\Delta_{f}$, the corresponding incremental steering angle (AFS) $\delta_{c}$, and the yaw torque $M_{z}$ are shown in Figure 3. In Figures 4 and 5 are given the controlled outputs $v_{y}$ and $\omega_{z}$ and its references $v_{y \text {,ref }}$ and $\omega_{z \text {,ref }}$ to track, and the tracking errors $v_{y}-v_{y \text {,ref }}$ and $\omega_{z}-\omega_{z \text {,ref }}$. The longitudinal velocity $v_{x}$ 


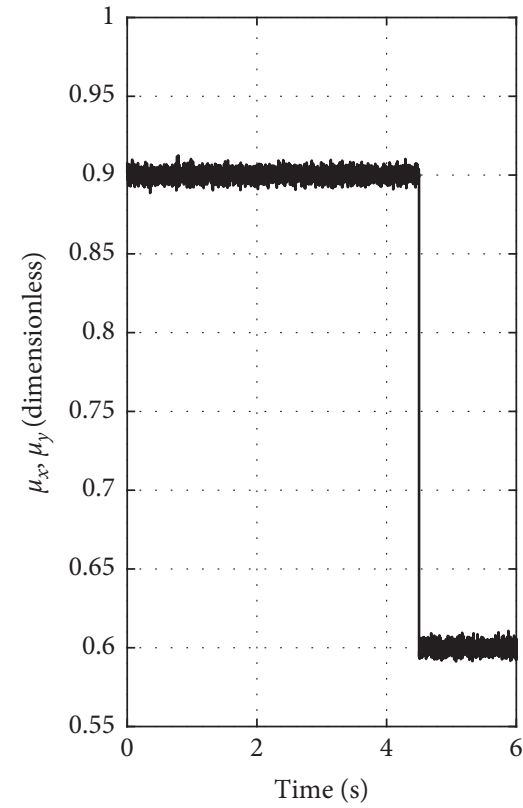

(a)

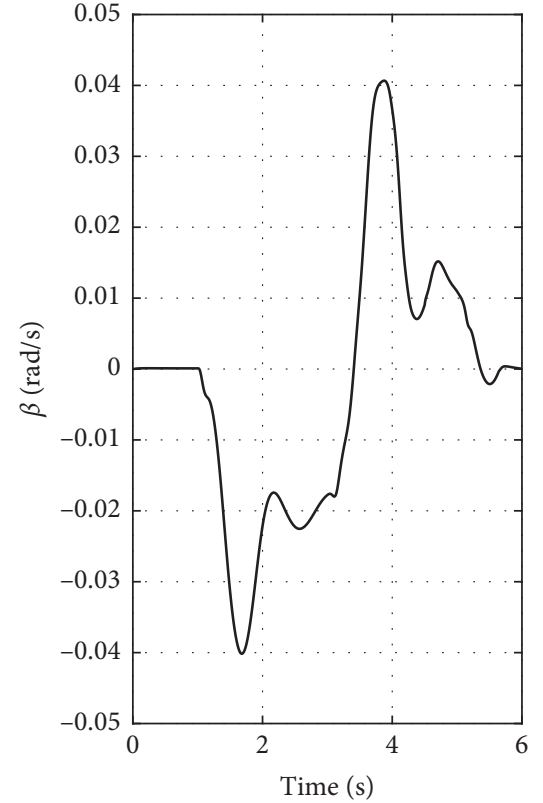

(b)

Figure 2: (a) Longitudinal/lateral tire-road friction coefficients $\mu_{x}$ and $\mu_{y}$; and (b) sideslip angle $\beta=\left(v_{y} / v_{x}\right)$.

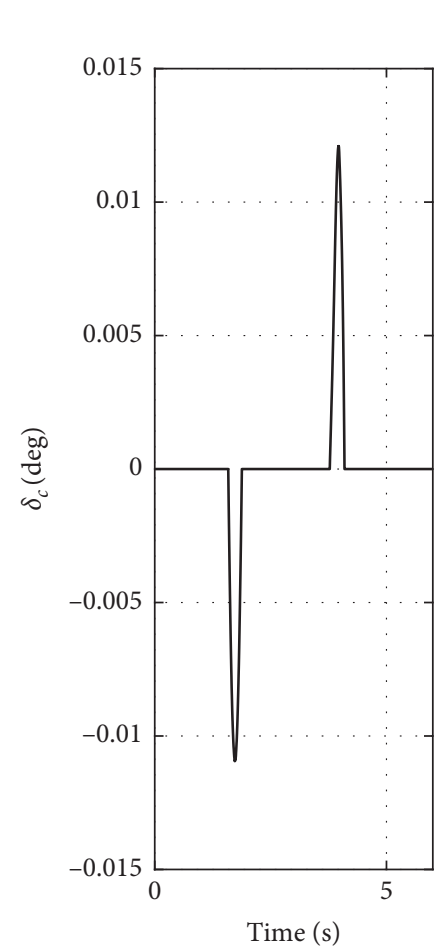

(a)

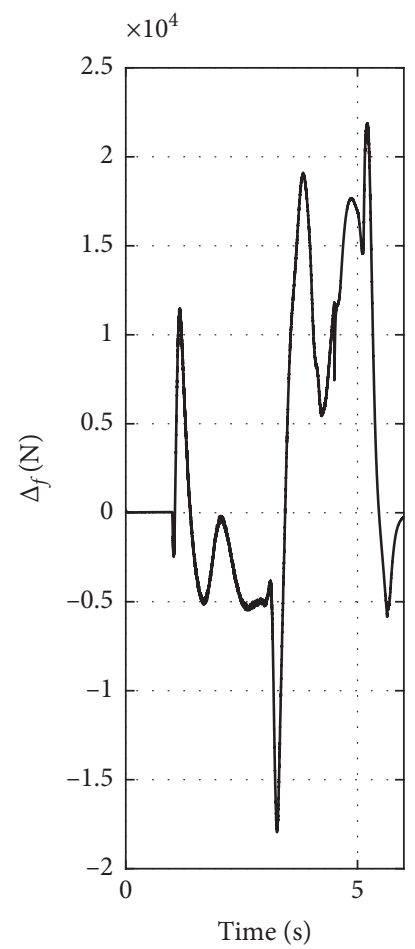

(b)

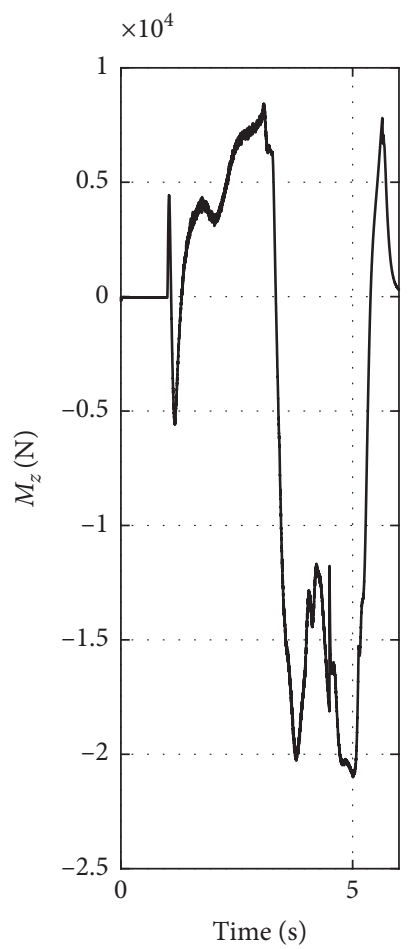

(c)

Figure 3: (a) Incremental steer angle $\delta_{c}$; (b) $\Delta_{f}$ controller; and (c) yaw momentum $M_{z}$. 


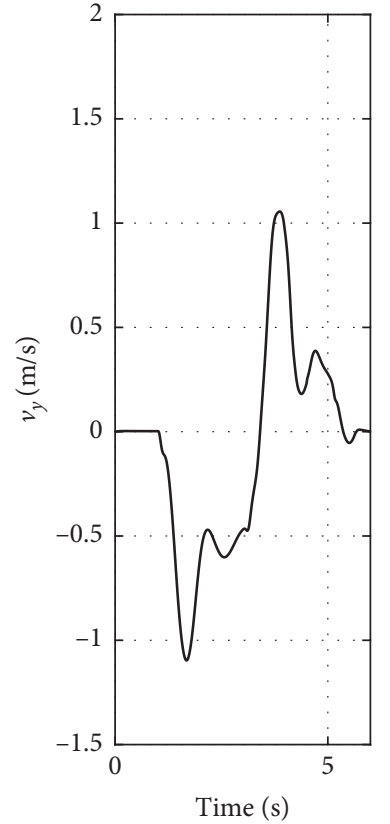

(a)

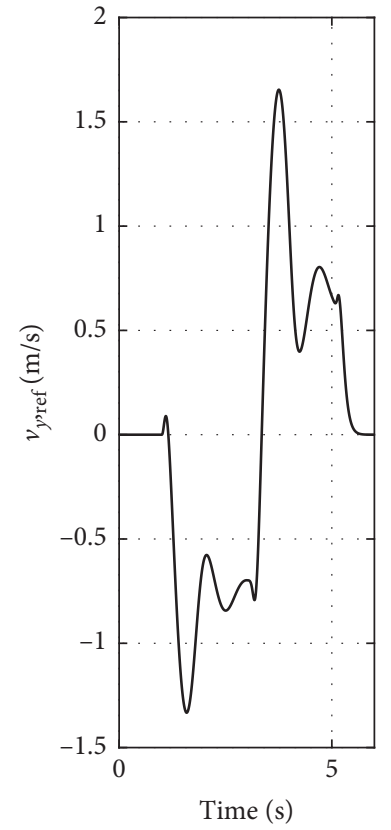

(b)

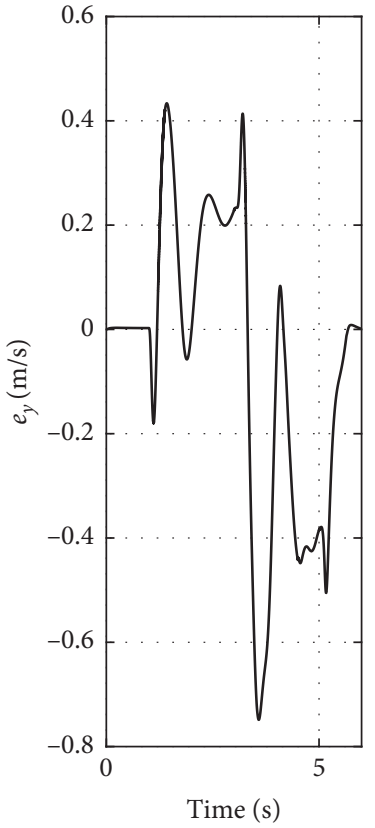

(c)

FIGURE 4: (a) Lateral velocity $v_{y}$; (b) lateral velocity reference $v_{y, \text { ref }}$; and (c) $e_{y}=v_{y}-v_{y, \text { ref }}$.

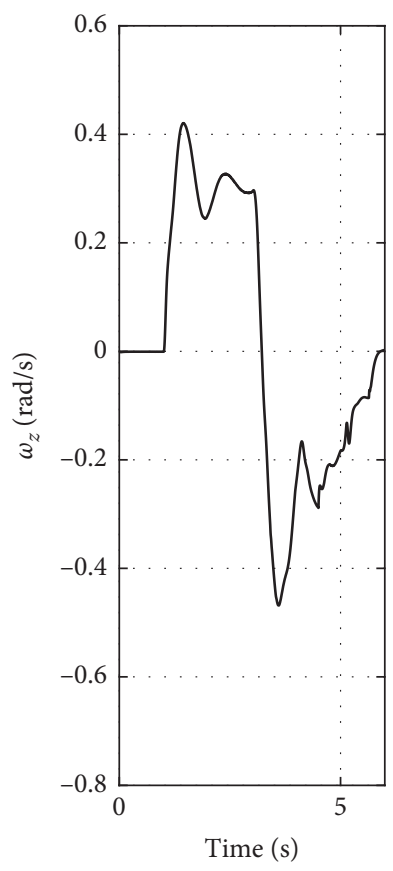

(a)

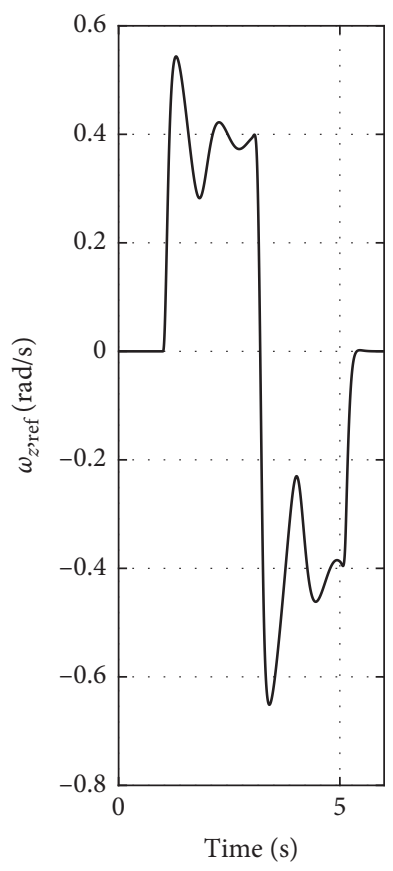

(b)

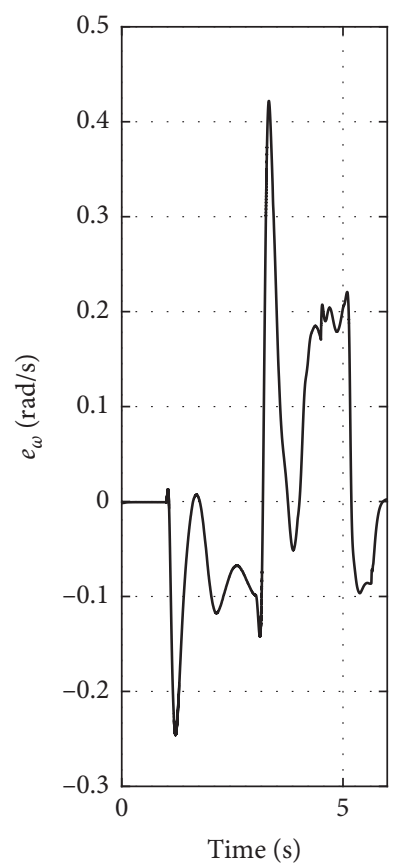

(c)

FIgURe 5: (a) Yaw rate $\omega_{z}$; (b) yaw rate reference $\omega_{z, \text { ref }}$; and (c) $e_{\omega}=\omega_{z}-\omega_{z \text {,ref }}$. 


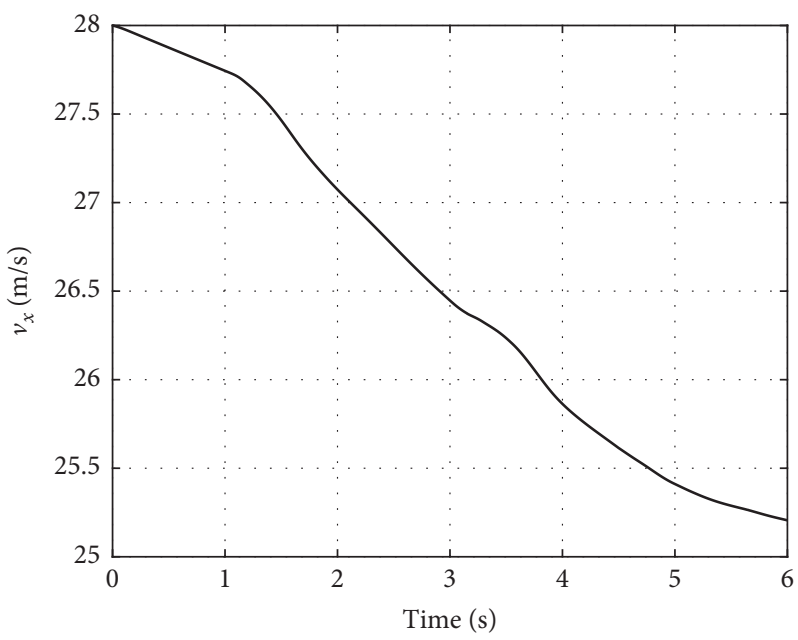

FIgURE 6: Longitudinal velocity $v_{x}$.

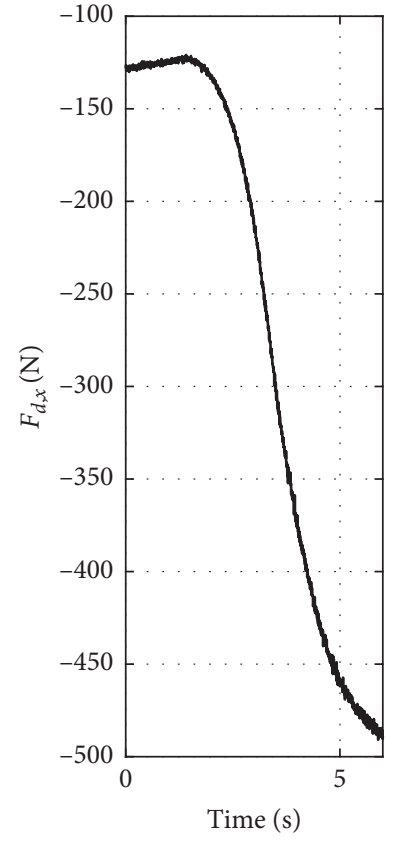

(a)

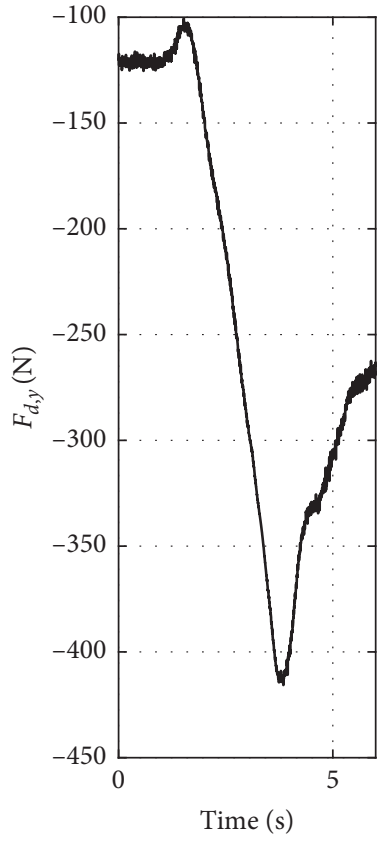

(b)

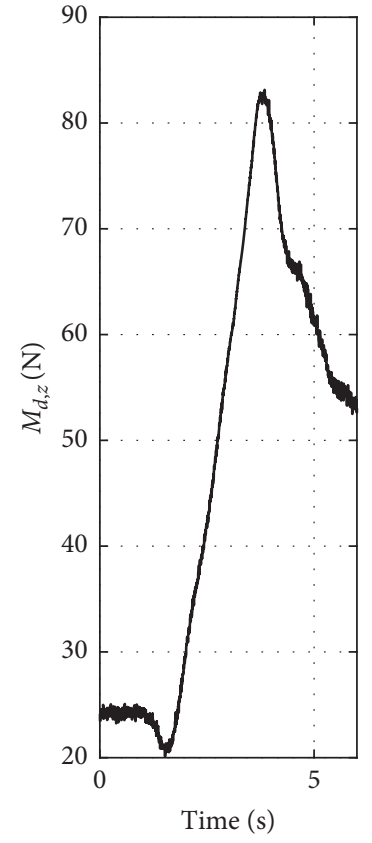

(c)

Figure 7: External disturbance: (a) $F_{d, x}$; (b) $F_{d, y}$; and (c) $M_{d, z}$.

is shown in Figure 6, and finally, the forces and moments $F_{d, x}, F_{d, y}, M_{d, z}$ are given in Figure 7.

\section{Conclusions}

In this study, an active front steering control and yaw moment was designed for the lateral and yaw dynamics of a vehicle. The active control of a robust nonlinear controller design uses the modified high-order sliding mode (HOSM) with the PI sliding surface. The stability performance of the PI sliding manifold was proved by the Lyapunov function. The simulation results highlight the performance of the proposed approach. The CarSim simulations show a good behavior of the HOSM-PI sliding surface even in the presence of external disturbances and parameter variations.

\section{Data Availability}

The data used to support the findings of this study are included within the article.

\section{Conflicts of Interest}

The authors declare that there are no conflicts of interest. 


\section{Acknowledgments}

This study was partially supported by the European Project ECSEL-JURIA-2018 "Comp4Drones" and by the Project "Coordination of autonomous unmanned vehicles for highly complex performances" and Executive Program of Scientific and Technological Agreement between Italy (Ministry of Foreign Affairs and International Cooperation) and Mexico (Mexican International Cooperation Agency for the Development), SAAP3.

\section{References}

[1] C. Acosta Lúa, B. Castillo-Toledo, R. Cespi, and S. Di Gennaro, "Tyre-road friction coefficient estimation based on tyre lateral deflection," in Proceedings of the 16th Congreso Latinoamericano de Control Automático - CLCA, pp. 1089-1094, Lima, Perú, October 2014.

[2] C. Acosta Lúa, B. Castillo Toledo, R. Cespi, and S. Di Gennaro, "An integrated active nonlinear controller for wheeled vehicles," Journal of the Franklin Institute, vol. 352, no. 11, pp. 4890-4910, 2015.

[3] C. A. Lua, B. Castillo-Toledo, R. Cespi, and S. Di Gennaro, "Nonlinear observer-based active control of ground vehicles with non negligible roll dynamics," International Journal of Control, Automation and Systems, vol. 14, no. 3, pp. 743-752, 2016.

[4] C. Acosta Lúa and S. Di Gennaro, "Nonlinear adaptive tracking for ground vehicles in the presence of lateral wind disturbance and parameter variations," Journal of the Franklin Institute, vol. 354, no. 7, pp. 2742-2768, 2017.

[5] X. Yi, R. Guo, and Y. Qi, "Stabilization of chaotic systems with both uncertainty and disturbance by the UDE-based control method," IEEE Access, vol. 8, no. 1, pp. 62471-62477, 2020.

[6] L. Liu, B. Li, and R. Guo, "Consensus control for networked manipulators with switched parameters and topologies," IEEE Access, vol. 9, pp. 9209-9217, 2021.

[7] G. Burgio, B. Castillo-Toledo, and S. Di Gennaro, "Nonlinear adaptive tracking for ground vehicles," in Proceedings of the 48th IEEE Conference on Decision and Control, pp. 7645-7650, Las Vegas, NV, USA, March 2009.

[8] A. Borri, D. Bianchi, M. D. Di Benedetto, and S. Di Gennaro, "Optimal workload actuator balancing and dynamic reference generation in active vehicle control," Journal of the Franklin Institute, vol. 354, no. 4, pp. 1722-1740, 2017.

[9] M. Mirzaei and H. Mirzaeinejad, "Fuzzy scheduled optimal control of integrated vehicle braking and steering systems," IEEE/ASME Transactions on Mechatronics, vol. 22, no. 5, pp. 2369-2379, 2017.

[10] A. N. Guzman, S. D. Gennaro, J. R. Dominguez, C. A. Lua, A. G. Loukianov, and B. Castillo-Toledo, "Enhanced discretetime modeling via variational integrators and digital controller design for ground vehicles," IEEE Transactions on Industrial Electronics, vol. 63, no. 10, pp. 6375-6385, 2016.

[11] Z. Sun, J. Zheng, Z. Man, H. Wang, and R. Lu, "Sliding mode-based active disturbance rejection control for vehicle steer-by-wire systems," IET Cyber-Physical Systems: Theory \& Applications, vol. 3, no. 1, pp. 1-10, 2018.

[12] H. Guo, F. Liu, F. Xu, H. Chen, D. Cao, and Y. Ji, "Nonlinear model predictive lateral stability control of active chassis for intelligent vehicles and its FPGA implementation," IEEE Transactions on Systems, Man, and Cybernetics: Systems, vol. 49, no. 1, pp. 2-13, 2019.
[13] Acosta Lúa C., Di Gennaro S., Navarrete Guzman A., Ortega Cisneros S., Rivera Domínguez J.. IEEE Transactions on Industrial Informatics 2019.

[14] L. Etienne, C. Acosta Lúa, S. Di Gennaro, and J. . -P. Barbot, "A Super-twisting controller for active control of ground vehicles with lateral tire-road friction estimation and carsim validation," International Journal of Control, Automation, and Systems, vol. 8, no. 5, pp. 1177-1189, 2020.

[15] C. Chen, J. Zhang, J. Du, and Y. Jia, "Non-linear decoupling control of vehicle plane motion," IET Control Theory \& Applications, vol. 6, no. 13, pp. 2083-2094, 2012.

[16] J. Tjonnas and T. A. Johansen, "Stabilization of automotive vehicles using active steering and adaptive brake control allocation," IEEE Transactions on Control Systems Technology, vol. 18, no. 3, pp. 545-558, 2010.

[17] D. Bianchi, A. Borri, M. D. D. Benedetto, S. D. Gennaro, and G. Burgio, "Adaptive integrated vehicle control using active front steering and rear torque vectoring," International Journal of Vehicle Autonomous Systems, vol. 8, pp. 85-105, 2010.

[18] S. V. Emelyanov, S. K. Korovin, and L. V. Levantovsky, "Higher order sliding regimes in the binary control systems," Soviet Physics, vol. 31, no. 4, pp. 291-293, 1986.

[19] L. Fridman, J. Moreno, and R. Iriarte, "Sliding modes after the first decade of the $21^{\text {st }}$ century: state of the art," Lecture Notes in Control and Information Sciences, Springer-Verlag Berlin, vol. 412, 2011.

[20] V. Utkin, A. Poznyak, Y. Orlov, and A. Polyakov, "Conventional and high order sliding mode control," Journal of the Franklin Institute, vol. 357, no. 15, pp. 10244-10261, 2020.

[21] V. Utkin, "Discussion aspects of high-order sliding mode control," IEEE Transactions on Automatic Control, vol. 61, pp. 829-833, 2015.

[22] J. Á. Mercado-Uribe and J. A. Moreno, "Discontinuous integral action for arbitrary relative degree in sliding-mode control," Automatica, vol. 118, Article ID 109018, 2020.

[23] X.-G. Guo and C. K. Ahn, "Adaptive fault-tolerant pseudoPID sliding-mode control for high-speed train with integral quadratic constraints and actuator saturation," IEEE Transactions on Intelligent Transportation Systems, vol. 24, pp. 1-11, 2020.

[24] U. Zakia, M. Moallem, and C. Menon, "PID-SMC controller for a 2-DOF planar robot," in Proceedings of the International Conference on Electrical, Computer and Communication Engineering, pp. 1-5, Kuala Lumpur, Malaysia, June 2019.

[25] G. Y. Gu, C.-X. Li, L.-M. Zhu, and S. Fatikow, "Robust tracking of nanopositioning stages using sliding mode control with a PID sliding surface," in Proceedings of the IEEE/ASME International Conference on Advanced Intelligent Mechatronics (AIM), pp. 973-977, Delft, The Netherlands, July 2014.

[26] I. Eker, "Second-order sliding mode control with PI sliding surface and experimental application to an electromechanical plant," Arabian Journal for Science and Engineering, vol. 37, 2012.

[27] A. H. Khan and S. Li, "Sliding mode control with PID sliding surface for active vibration damping of pneumatically actuated soft robots," IEEE Access, vol. 8, pp. 88793-88800, 2020.

[28] M. Labbadi and M. Cherkaoui, "Novel robust super twisting integral sliding mode controller for a quadrotor under external disturbances," International Journal of Dynamics and Control, vol. 8, no. 3, pp. 805-815, 2020.

[29] G. J. Heydinger, W. R. Garrott, J. P. Chirstos, and D. A. Guenther, A Methodology for Validating Vehicle 
Dynamics Simulations, Society of Automotive Engineers, Warrendale, PA, USA, Paper 900128, 1990.

[30] J. Wong, Theory of Ground Vehicles 1978, Wiley, New York, NY, USA.

[31] P. Setlur, J. R. Wagner, D. M. Dawson, and D. Braganza, "A trajectory tracking steer-by-wire control system for ground vehicles," IEEE Transactions on Vehicular Technology, vol. 55, no. 1, pp. 76-85, 2006.

[32] G. Burgio and P. Zeglaar, "Integrated vehicle control using steering and brakes," International Journal of Control, vol. 79, no. 2, pp. 162-169, 2006.

[33] H. Pacejka, Tyre and Vehicle Dynamics, Elsevier Butterworth-Heinemann, Oxford, UK, 2005.

[34] O. Hanke, T. Bertram, and M. Hiller, "Analysis and control of vehicle dynamics under crosswind conditions," in Proceedings of the 2001 IEEE/ASME International Conference on Advanced Intelligent Mechatronics, pp. 331-336, Como, Italy, July 2001. 